\title{
Convective Difference Schemes
}

\author{
By K. V. Roberts and N. O. Weiss
}

1. Introduction. In this paper general methods are developed for numerical solution of the partial differential equations for the convection of a scalar (e.g. density) or a vector (e.g. magnetic field). The difference schemes are correctly centred in both space and time so that the modulus of the amplification factor is exactly unity. They are also conservative and have fourth order accuracy. The methods are applicable in two or three space dimensions and on curvilinear as well as Cartesian meshes. They can be used for either linear or nonlinear problems.

We consider finite difference schemes for the numerical solution of the hyperbolic partial differential equations

$$
\frac{\partial \rho}{\partial t}=-\operatorname{div} \mathbf{F}
$$

and

$$
\frac{\partial \mathbf{B}}{\partial t}=-\operatorname{curl} \mathbf{E} \text {. }
$$

The dependent variables can be represented on a fixed Eulerian net with characteristic spacing $\Delta r$ and we aim to establish explicit methods that are accurate in three space dimensions. In general, the total machine time required for a problem then varies ${ }^{1}$ as $(\Delta r)^{-4}$; it is therefore important to devise a method of solution that is both efficient and precise. The numerical techniques have been developed for solving magnetohydrodynamic problems in three dimensions and examples are taken from this context. However, the methods may be generally applied. Their development is facilitated by adopting a physical approach.

More specifically, we consider the Eulerian equations

$$
\frac{\partial \rho}{\partial t}=-\nabla \cdot\left(\rho \mathfrak{u}-\eta_{1} \boldsymbol{\nabla} \rho\right)
$$

and

$$
\frac{\partial \mathbf{B}}{\partial t}=\boldsymbol{\nabla} \wedge\left(\mathbf{u} \wedge \mathbf{B}-\eta_{2} \boldsymbol{\nabla} \wedge \mathbf{B}\right)
$$

for the convection of scalar and vector quantities (density and magnetic field) by a velocity field $\mathfrak{u}$ with magnitude $U$ in a region of dimension $L$, where the diffusion coefficients $\eta_{1}$ and $\eta_{2}$ are both small (i.e. the Péclet number, $U L / \eta_{1}$, and the magnetic Reynolds number, $U L / \eta_{2}$, are both large). The vector equation (1.4) must be solved subject to the condition

$$
\operatorname{div} \mathbf{B}=\mathbf{0}
$$

and in many problems the flow can be regarded as incompressible, so that

$$
\operatorname{div} \mathbf{u}=0 .
$$

Received March 11, 1965.

1 We assume that diffusion is weak compared with convection, so that the equations are essentially hyperbolic. If they were parabolic, the total computing time would vary as $\Delta r^{-5}$ and it would be advisable to use implicit methods. 
We insist that magnetic flux must be conserved exactly throughout the finite difference process. Where (1.6) is applicable, its difference analogue has similarly to be satisfied.

The difference schemes are first illustrated by considering Equation (1.3) in one dimension, when (1.6) implies that $\mathfrak{u}$ is constant. The problem is then linear and its treatment is consequently simple. Stability and accuracy are discussed in $\$ \S 2-4$ where we emphasize the importance of choosing schemes that are correctly centred in both space and time. $\$ \$ 5$ and 6 contain a detailed discussion of methods for solving (1.3) and (1.4) in two dimensions and, finally, we indicate in $\$ 8$ how three dimensional problems might be tackled. The methods described are suitable for solving nonlinear problems (in which $\mathbf{u}$ also is a dependent variable) but solution of the equation of motion must depend on details of the particular physical system and this will not be discussed here.

Equations such as (1.1) and (1.2) are described as conservation laws [1, 2]. The continuity equation states that the increase of the mass within a given volume is equal to the total flux of matter into that volume, while Faraday's law equates the rate of change of magnetic flux across a surface to the integral of the electric field along a line bounding that surface. For finite difference approximations the integral formulations

$$
\Delta \int \rho d \tau=-\iint \mathbf{F} \cdot d \mathbf{S} d t
$$

and

$$
\Delta \int \mathbf{B} \cdot d \mathbf{S}=-\int \oint \mathbf{E} \cdot d \mathbf{l} d t
$$

of the conservation laws are more convenient than the differential equations (1.1) and (1.2). We therefore regard these equations as integrated over elements of volume or area defined by mesh points: thus the value of $\rho$ corresponding to a given point on the mesh represents the average density of matter within a box surrounding that point. Each component of $\mathbf{B}$ likewise represents the magnetic flux across an area normal to that component and centred on the point. Similarly, F or E must be defined as double averages, over elements of surfaces or along lines respectively, as well as over a time interval $\Delta t$. The finite difference scheme must then itself be conservative: that is, Equations (1.7) and (1.8) must apply exactly to all regions that can be built up from the basic elements mentioned above. Only such schemes are acceptable and we furthermore require methods that have fourth order accuracy.

Errors in finite difference processes are mainly caused by the presence of Fourier modes with wave lengths of only a few mesh intervals. Moreover, in a practical computation it is cheaper to reduce $\Delta t$ than it is to shorten $\Delta r$. We therefore devise schemes in which the error is of fourth order in $\Delta r$ but only of second order in $\Delta t$. But the accuracy is not impaired so long as

$$
\left(\frac{U \Delta t}{\Delta r}\right)^{2} \ll 1
$$

and it is sufficient to have $(U \Delta t / \Delta r) \lesssim 1 / 4$. 
The integral formulation of the equations simplifies the development of these schemes. In addition, it gives a precise meaning to conservation. When the velocity $\mathbf{u}$ is constant, integral and differential formulations lead to the same difference formulae. If $\mathbf{u}$ is a function of position, the two approaches diverge for a scalar and differ entirely for the vector field $\mathbf{B}$.

The treatment of diffusion when $\eta_{1}$ and $\eta_{2}$ are small is discussed in $\$ 7$. To a close approximation the density and magnetic field are then convected with the fluid. Numerical errors inherent in the treatment of the convective operator $(\mathbf{u} \cdot \boldsymbol{\nabla})$ could be avoided by adopting a moving Lagrangian mesh. In one dimension, this is nearly always the best approach. For coupled magnetohydrodynamic equations in several dimensions, the mesh distortion is so severe that Eulerian schemes may be preferable. Conservation can be applied to either type of mesh, for example, Faraday's law (1.8) may be expressed either in fixed or in moving co-ordinates.

The general discussion is presented in terms of Cartesian coordinate systems. The transition to polar co-ordinates is straightforward and is considered in the penultimate section. Our emphasis throughout is on the relationship of the physics to the numerical procedures and on the choice of practical meshes and difference schemes. We hope that the methods described will prove useful in tackling real problems. The notation demands many suffixes but we have endeavoured to keep it consistent.

2. Centred Difference Schemes in One Dimension. The prime requirement of a difference scheme is that it should be stable; we develop schemes that are also conservative and free of numerical dissipation and whose truncation errors are of fourth order in the mesh interval. These distinctions are best illustrated by considering the one dimensional convective equation

$$
\frac{\partial \rho}{\partial t}=-u \frac{\partial \rho}{\partial x}
$$

with $u$ constant, whose trivial solution is

$$
\rho(x, T+t)=\rho(x-u t, T) .
$$

(This problem merely serves as a model for multidimensional Eulerian calculations; in practice, (2.1) should be integrated along characteristics, i.e. using a Lagrangian mesh.) In this section we discuss stability and numerical damping and demonstrate the advantages of difference schemes that are correctly centred in both space and time.

Let $\rho$ be defined by values $\rho_{j}{ }^{n}$ at mesh points in the $x-t$ plane with co-ordinates $\left(x_{j}, t^{n}\right)=(j \Delta x, n \Delta t)$ where $j, n$ are integers. The values of $\rho_{j}{ }^{n+1}$ are calculated from $\rho_{j}{ }^{n}$ by some difference scheme which approximates to (2.1). The error is

$$
\epsilon_{j}{ }^{n}=\rho_{j}{ }^{n}-\rho(j \Delta x, n \Delta t) .
$$

Usually, $\Delta t$ and $\Delta x$ are of the same order ${ }^{2}$ and the difference scheme is said to be accurate of order $p$ if after a single time step

$$
\left|\epsilon_{j}^{\prime}\right| \leqq O\left(\Delta t^{p+1}\right)
$$

${ }^{2}$ When $\Delta x \gg u \Delta t$ it is more appropriate to express the error in terms of the mesh interval (see \$4). 
It is evident from (2.2) that the evaluation of $\rho_{j}{ }^{n+1}$ can be regarded as an interpolation problem. Linear interpolation between $\rho_{j-1}^{n}$ and $\rho_{j+1}^{n}$ yields the most obvious Eulerian approximation to (2.1). The derivatives are replaced by first differences and $\partial \rho / \partial x$ is estimated at the initial time $t^{n}$ :

$$
\rho_{j}^{n+1}=\rho_{j}^{n}-\frac{1}{2} \mu\left(\rho_{j+1}^{n}-\rho_{j-1}^{n}\right)
$$

where

$$
\mu=\frac{u \Delta t}{\Delta x} .
$$

This scheme has first order accuracy but is well known to be unstable; to first order, it is in fact an approximation to

$$
\frac{\partial \rho}{\partial t}=-u \frac{\partial \rho}{\partial x}-\frac{1}{2} u^{2} \Delta t \frac{\partial^{2} \rho}{\partial x^{2}}
$$

whose solutions grow exponentially with time. This is because $\partial \rho / \partial x$ has been estimated at $t^{n}$ instead of being centred, like the time difference, at $t^{n+1 / 2}$.

The simplest expedient for preventing instability is to interpolate between two adjacent points at the original time level. This leads to the "one-sided" difference scheme, originally suggested by Lelevier [3], in which (2.1) is represented by

$$
\rho_{j}^{n+1}=\rho_{j-l}^{n}-\mu^{\prime}\left(\rho_{j-l}^{n}-\rho_{j-l-1}^{n}\right) .
$$

where $l$ is chosen so that $l \leqq \mu<l+1$ and $\mu^{\prime}=\mu-l$. In this form, the scheme is unconditionally stable and has first order accuracy; for $|\mu| \leqq 1$ it approximates to the differential equation

$$
\frac{\partial \rho}{\partial t}=-u \frac{\partial \rho}{\partial x}+\frac{1}{2}|u| \Delta x(1-|\mu|) \frac{\partial^{2} \rho}{\partial x^{2}} .
$$

The instability associated with (2.7) is now masked by a "numerical diffusion" whose effects are easily demonstrated by making a Fourier transform of (2.8) and taking the component $\rho(k)$ with wave number $k$. (The range of $k$ is $\pi / J \Delta x \leqq$ $k \leqq \pi / \Delta x$, where $J$ is the total number of mesh intervals in the $x$-direction.) We define the amplification factor $\lambda(k)$ by

$$
\rho^{n+1}(k)=\lambda(k) \rho^{n}(k)
$$

and the von Neumann condition for stability [3] is that

$$
|\lambda| \leqq 1+O(\Delta t) \text {. }
$$

For the one-sided difference scheme,

$$
|\lambda|^{2}=1-4 \mu^{\prime}\left(1-\mu^{\prime}\right) \sin ^{2}\left(\frac{1}{2} k \Delta x\right)
$$

and this stability criterion is satisfied. Now the true solution of (2.1) has

$$
\lambda=\exp (-i \mu k \Delta x)
$$

with $|\lambda|=1$ and the numerical error, which produces anomalous damping, is apparent from Table 1. (Error in the argument of $\lambda$ leads to dispersion, which will be discussed in §3.) The approximation improves as $|\mu|$ and $k \Delta x$ tend to zero but 
TABLE 1

Damping in First and Second Order Schemes

\begin{tabular}{|c|c|c|c|c|c|c|}
\hline \multirow{3}{*}{$k \Delta x$} & \multicolumn{3}{|c|}{ One-sided } & \multicolumn{3}{|c|}{ Three-point } \\
\hline & \multicolumn{3}{|c|}{$\mu$} & \multicolumn{3}{|c|}{$\mu$} \\
\hline & 0.05 & 0.25 & 0.5 & 0.05 & 0.25 & 0.5 \\
\hline $18^{\circ}$ & .9977 & .9908 & .9877 & 1.0000 & .9999 & .9998 \\
\hline $30^{\circ}$ & .9936 & .9746 & .9659 & 1.0000 & .9995 & .9983 \\
\hline $45^{\circ}$ & .9860 & .9435 & .9239 & .9999 & .9975 & .9919 \\
\hline $60^{\circ}$ & .9760 & .9014 & .8660 & .9997 & .9926 & .9763 \\
\hline $90^{\circ}$ & .9513 & .7906 & .7071 & .9988 & .9703 & .9014 \\
\hline $120^{\circ}$ & .9260 & .6614 & .5000 & .9972 & .9318 & .7603 \\
\hline $180^{\circ}$ & .9000 & .5000 & 0 & .9950 & .8750 & .5000 \\
\hline
\end{tabular}

the finite differences are naturally inadequate for wave lengths close to the limit $2 \Delta x$. The major weakness of this difference scheme lies, of course, in the strong numerical damping. Since the scheme is accurate only to first order, dissipation remains important even in the limit $\Delta t \rightarrow 0$; the number of steps per unit time, $N$, becomes infinite and the total amplification factor

$$
|\lambda|^{N} \rightarrow \exp -\left(\frac{2 u \sin ^{2}\left(\frac{1}{2} k \Delta x\right)}{\Delta x}\right) .
$$

The strength of this damping is clear from Figure 1, which shows the total amplification factor after a mode has been transported through a distance $\Delta x$, plotted on a polar diagram as a function of $k \Delta x$. Actual damping of a humped profile is illustrated in Figure 2(a). Thus first order schemes are inadequate if accuracy is required. $^{3}$

Three point interpolation allows second order accuracy. The simplest such scheme is

$$
\rho_{j}^{n+1}=\rho_{j}^{n}-\frac{1}{2} \mu\left[(1-\mu) \rho_{j+1}^{n}+2 \mu \rho_{j}^{n}-(1+\mu) \rho_{j-1}^{n}\right]
$$

for which

$$
|\lambda|^{2}=1-4 \mu^{2}\left(1-\mu^{2}\right) \sin ^{4}\left(\frac{1}{2} k \Delta x\right) .
$$

This is stable for $|\mu| \leqq 1$; numerical damping is still present, as is shown in Table 1 and Figure 2(b), but this becomes insignificant as $\mu \rightarrow 0$, when

$$
|\lambda|^{N}=\left[1-O\left(\mu^{2}\right)\right]^{N} \rightarrow 1 .
$$

This scheme is equivalent to using equation (2.1) itself to provide an approximation to the time-centred spatial derivative by writing

$$
\left(\frac{\partial \rho}{\partial x}\right)^{n+1 / 2}=\left(\frac{\partial \rho}{\partial x}\right)^{n}+\frac{1}{2} \Delta t\left(\frac{\partial^{2} \rho}{\partial x \partial t}\right)^{n}=\left(\frac{\partial \rho}{\partial x}\right)^{n}-\frac{1}{2} u \Delta t\left(\frac{\partial^{2} \rho}{\partial x^{2}}\right)^{n}
$$

3 Nevertheless, the one-sided scheme preserves the sign of positive definite quantities, as do Lagrangian methods also; this property is not shared by space-centred Eulerian schemes. 


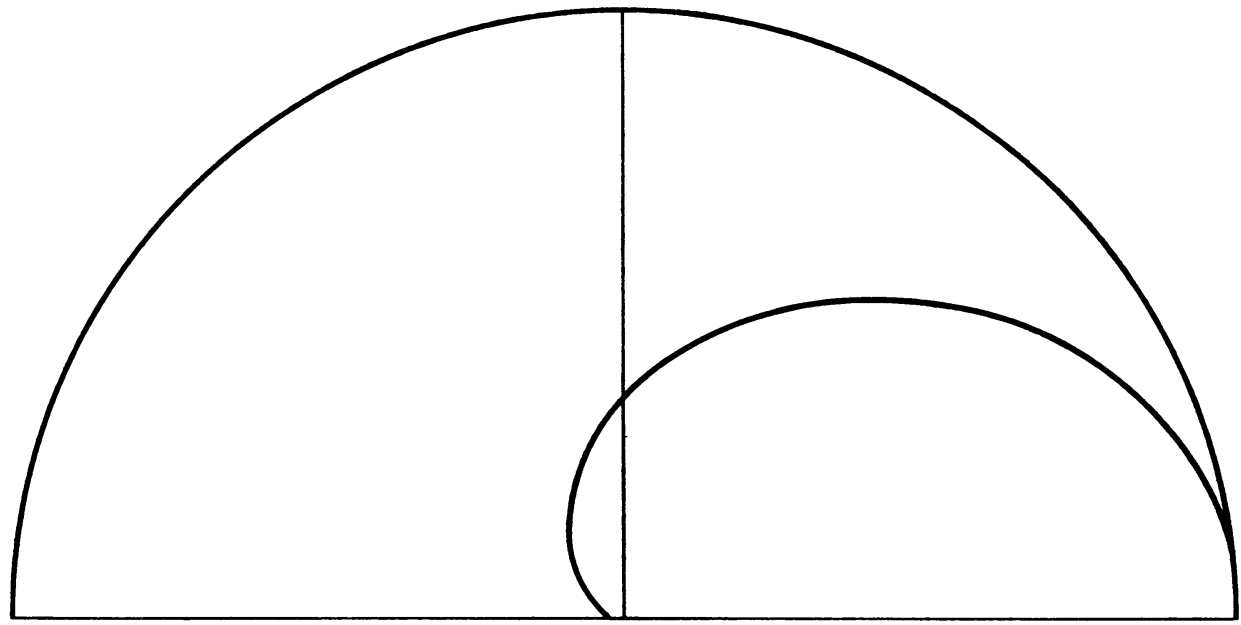

FIGURE 1. One-sided derivatives: numerical damping as $\Delta t \rightarrow 0$. The modulus of the total amplification factor after a time $\Delta x / u$ (so that each mode should have been transported through one mesh interval) is plotted on a polar diagram as a function of the angle $k \Delta x$.
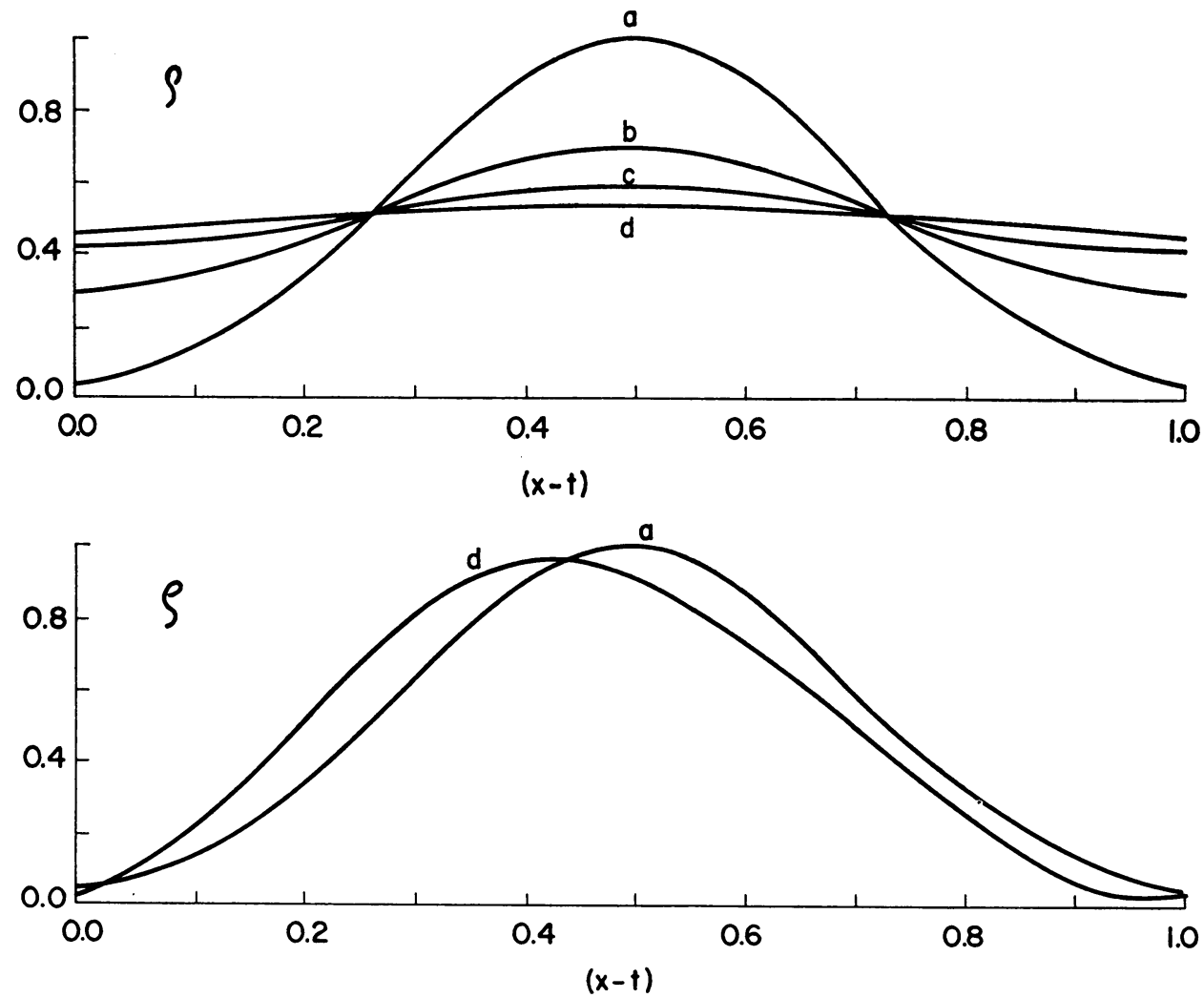

Figure 2. Numerical damping in first and second order schemes. The convection of a Gaussian profile with unit velocity on a mesh with $\Delta x=1 / 20, \mu=0.125$, subject to periodic boundary conditions. Curves $a, b, c, d$ show profiles at $t=0.0,1.0,2.0,3.0$ respectively. (a) One-sided derivatives. (b) Three-point method. 
This method was introduced by Lax and Wendroff [4], [5] and has been extensively used and developed. Numerical damping is still present but this is sometimes advantageous in eliminating unwanted high wave number modes. On the other hand, the method can become very complicated with equations less simple than (2.1). ${ }^{4}$ Moreover, the physical significance and the magnitude of the diffusion may be difficult to assess. We prefer to exclude dissipation from the convective equation altogether, by making $|\lambda|$ identically unity, and to include a separate diffusion term explicitly when this proves necessary (see \$7). A similar approach has been adopted by Kreiss [7].

Schemes with $|\lambda|=1$ are readily devised. It is sufficient that all differences should be correctly centred in space and time about the point $\left(x_{j}, t^{n+1 / 2}\right)$. Such a scheme can be built up on two time levels by combining terms to form a difference equation

$$
\sum_{l} a_{l}\left(\rho_{j-l}^{n+1}-\rho_{j+l}^{n}\right)=0
$$

with real coefficients $a_{l}$. The amplification factor then satisfies

$$
\lambda \sum a_{l} e^{-i l \theta}-\sum a_{l} e^{i l \theta}=0
$$

where

$$
\theta=k \Delta x
$$

This has the form

$$
A \lambda-A^{*}=0
$$

(where $A^{*}$ denotes the complex conjugate of $A$ ) so that $|\lambda|=1$ for all values of $\mu$ and $\theta$. The conditions under which it is necessary to adopt a scheme of the form (2.19) will be discussed elsewhere.

3. Nondissipative Difference Schemes with Second and Fourth Order Accuracy. We have shown that it is possible to devise difference schemes that are correctly centred in both space and time and for which $|\lambda|$ is identically equal to unity. Moreover, these schemes can be expressed in conservative form (see $\$ 4$ ). We give two explicit second order methods in this section. Their solutions converge to that of the differential equation as $\Delta x \rightarrow 0$. But this limit cannot be attained in practice. In three space dimensions the total computing time varies inversely as the fourth power of the mesh spacing $\Delta r$. The minimum possible space interval, $\Delta r_{0}$, thus varies inversely only as the fourth root of the available machine time (or the programming efficiency or computer speed). These latter would have to be increased by a factor $10^{4}$ merely to reduce $\Delta r_{0}$ by 10 . For practical purposes, therefore, we have to regard $\Delta r_{0}$ as a fixed quantity and to devise methods which then achieve the maximum accuracy. We therefore describe two schemes with fourth order accuracy. These schemes are stable provided that the Courant-FriedrichsLewy criterion is satisfied, i.e. so long as the domain of dependence of the difference equation includes that of the differential equation itself.

${ }^{4}$ The Lax-Wendroff method is easier to apply if it is split into two steps [6] but the same accuracy cannot be obtained without halving the mesh interval in each dimension. 
Angled derivative scheme. Suppose the integration sweeps in the direction of increasing $x$ for each value of $t$. When we are about to calculate $\rho_{j}{ }^{n+1}$ the values of $\rho_{k}{ }^{n}(1 \leqq k \leqq j)$ and $\rho_{k}{ }^{n+1}(1 \leqq k \leqq j-1)$ are available. Thus we can use the "angled derivative" centred on $\left(x_{j}, t^{n+1 / 2}\right)$ to produce a difference scheme with second order accuracy [8]. This can be written (see Figure 3(a))

$$
\rho_{j}{ }^{n+1}=\rho_{j}{ }^{n}-\xi\left(\rho_{j+1}^{n}-\rho_{j-1}^{n+1}\right)
$$

where

$$
\xi=\frac{\frac{1}{2} \mu}{1+\frac{1}{2} \mu} .
$$

Only the latest value of $\rho$ available at each point appears in $(3.1) .^{5}$ The value of the amplification factor depends on the sign of $\mu$ and it is generally necessary to alternate the direction of integration with each time step so that the resultant value of $\lambda$ is the geometric mean of $\lambda(\mu)$ and $\lambda(-\mu)$.

The domain of dependence of (3.1) includes all the points from $x_{1}$ to $x_{j}$ at time $t^{n+1}$ but only $x_{j}$ and $x_{j+1}$ at the original time level. Thus the proof that $|\lambda|=1$ for all $\mu$ in $\$ 2$ appears to contradict the Courant-Friedrichs-Lewy criterion when $\mu<-1$. This paradox can be resolved: for (3.1) must in general be regarded as implicit if the boundary conditions are to be satisfied. Even so, any error either in setting the boundary value or in calculating an interior point will be magnified as the sweep proceeds if $|\xi|>1$. In order to avoid the spatial amplification of rounding errors it is necessary that the influence of a point should diminish as the distance from it increases. For the angled derivative scheme this imposes the condition

$$
-1 \leqq \mu \lesssim 2
$$

which has been verified by numerical tests.

Staggered mesh. An alternative approach, originally suggested by von Neumann, uses a staggered mesh [11] (see Figure 3(b)). If $\rho_{j}$ is defined at $t^{n}, t^{n+1}$, then its neighbours $\rho_{j_{ \pm 1}}$ are defined at the intermediate level $t^{n+1 / 2}$. Any space difference on this level is automatically centred in time. The derivative $\partial \rho / \partial x$ can be represented by an arbitrary combination of differences at integral and half-integral time levels. The modulus of the amplification factor will be exactly unity if all the differences are correctly centred in both space and time: this means that the difference equation takes the form

$$
\sum_{l} a_{l}\left(\rho_{j-2 l}^{n+1}-\rho_{j+2 l}^{n}\right)+\sum_{l} b_{l}\left(\rho_{j-2 l+1}^{n+1 / 2}-\rho_{j+2 l-1}^{n+1 / 2}\right)=0 .
$$

For $\lambda$ then satisfies

$$
A \lambda+2 i B \lambda^{1 / 2}-A^{*}=0
$$

where

$$
A=\sum_{l} a_{l} e^{-2 i l \theta} \text { and } B=\sum_{l} b_{l} \sin (2 l-1) \theta
$$

Then

$$
\lambda^{1 / 2}=-\frac{\left[i B \pm\left(A A^{*}-B^{2}\right)^{1 / 2}\right]}{A}
$$

\footnotetext{
${ }^{5}$ A similar method was suggested by Saul'ev for solving the diffusion equation [9], [10].
} 


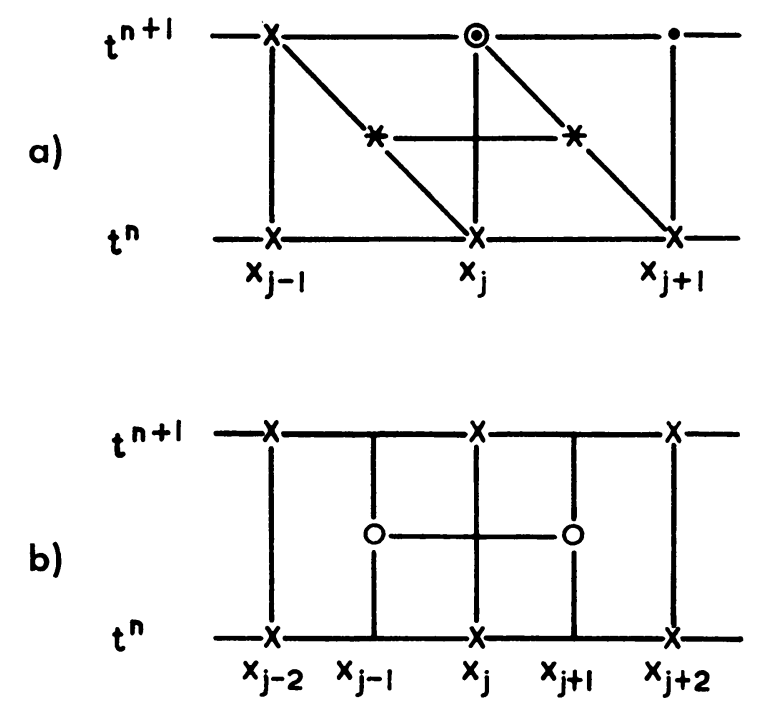

FIGURE 3. Conservative difference schemes with second order accuracy. (a) Angled derivatives. We know values of $\rho$ at the points denoted by crosses in the $x-t$ plane and, implicitly, at $\left(x_{j}, t^{n+1}\right)$. These values provide estimates of $\rho$ at the points marked by asterisks, which in turn give a difference which is properly centred in both $x$ and $t$. (b) Staggered mesh. Here $\rho$ is calculated at the points where its value is required, leading to a mesh staggered in space and time.

and $|\lambda|=1$ provided that

$$
B^{2} \leqq A A^{*} .
$$

Three-level schemes (unlike the two-level ones discussed in \$2) are only conditionally stable.

The simplest scheme is

$$
\rho_{j}^{n+1}=\rho_{j}^{n}-\frac{1}{2} \mu\left(\rho_{j+1}^{n+1 / 2}-\rho_{j-1}^{n+1 / 2}\right) .
$$

This has second order accuracy and $|\lambda|=1$ for $|\mu| \leqq 2$.

After one time step, the phase of a Fourier component should change by an amount

$$
\phi_{0}=-\frac{u \Delta t}{\Delta x} \cdot k \Delta x=-\mu \theta .
$$

The corresponding phase shift for the angled derivative method is

$$
\phi_{1}=-\sin ^{-1} \frac{\mu \sin \theta\left[1+\frac{1}{2} \mu(1-\cos \theta)\right]}{1+\frac{1}{2} \mu^{2}(1-\cos \theta)}
$$

while that for the staggered scheme is

$$
\phi_{2}= \pm 2 \sin ^{-1}\left(\frac{1}{2} \mu \sin \theta\right) .
$$

There are two solutions to (3.9), one of which represents a disturbance travelling in the wrong direction which is only eliminated if the initial values are correctly set on both time levels [12]. As $\mu \rightarrow 0$, the ratios $\phi_{1} / \phi_{0}$ and $\phi_{2} / \phi_{0}$ both tend to $\sin \theta / \theta$, 
TABLE 2

Velocity Dispersion

\begin{tabular}{c|c|c|c}
\hline \multirow{2}{*}{$k \Delta X$} & Second order & \multicolumn{2}{|c}{ Fourth order } \\
\cline { 2 - 3 } & $\phi_{2} / \phi_{0}$ & $\phi_{3} / \phi_{0}$ & $\phi_{4} / \phi_{0}$ \\
\hline $18^{\circ}$ & .9836 & .9992 & .9997 \\
$30^{\circ}$ & .9549 & .9947 & .9976 \\
$45^{\circ}$ & .9003 & .9753 & .9883 \\
$60^{\circ}$ & .8270 & .9304 & .9648 \\
$90^{\circ}$ & .6366 & .7427 & .8489 \\
$120^{\circ}$ & .4135 & .4652 & .6203 \\
$180^{\circ}$ & 0 & 0 & 0 \\
\hline
\end{tabular}

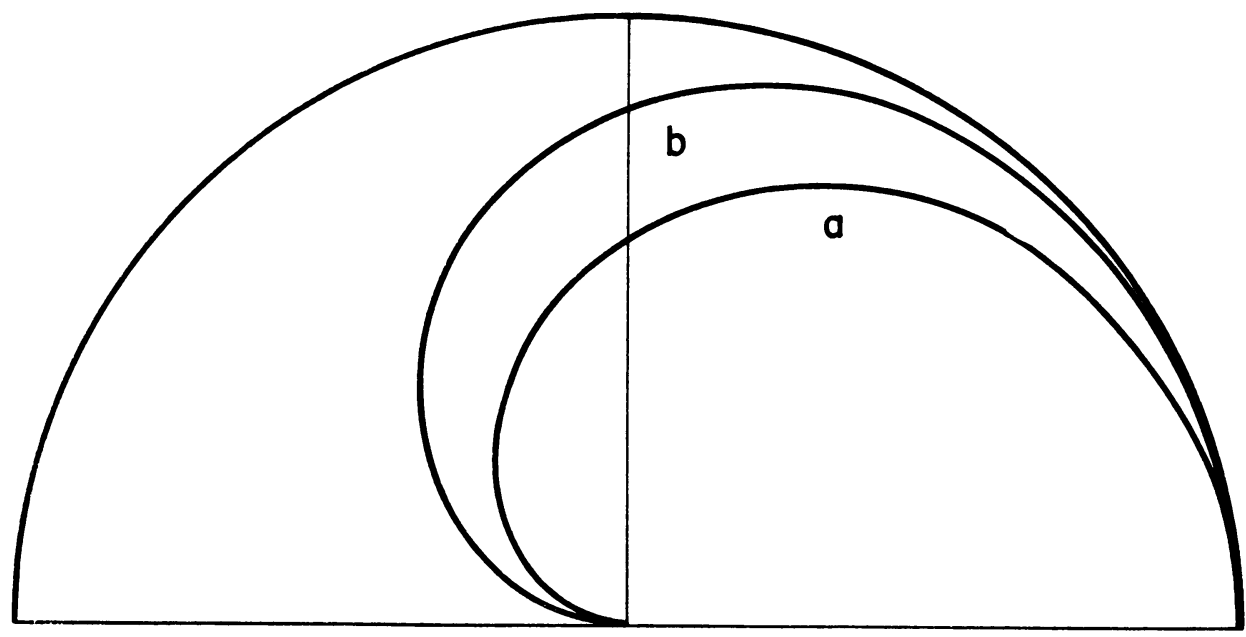

FIGURE 4. Velocity dispersion in the limit $\mu \rightarrow 0$ for second and fourth order schemes. The ratios $\phi_{2} / \phi_{0}(a)$ and $\phi_{4} / \phi_{0}(b)$ are plotted on a polar diagram as functions of the angle $k \Delta x$.

which is listed in the first column of Table 2 and displayed in Figure 4. The shortest wave length components $(\theta=\pi)$ do not move at all and even the wave length $4 \Delta x$ has only two-thirds of its proper speed, although the longest wave lengths move with nearly the correct velocity. This dispersion at high wave numbers is the most serious error remaining in the difference schemes and its effects are illustrated in Figure 5(a). To diminish them, it is necessary to develop approximations that possess fourth order accuracy.

Fourth order scheme on staggered mesh. The simplest fourth order scheme uses four values of $\rho$ at $t^{n+1 / 2}$ to estimate the spatial derivative:

$$
\begin{aligned}
\rho_{j}^{n+1}=\rho_{j}^{n}-\frac{1}{2} \mu & {\left[\left(\rho_{j+1}^{n+1 / 2}-\rho_{j-1}^{n+1 / 2}\right)\right.} \\
& \left.+\frac{1}{24}\left(1-\frac{1}{4} \mu^{2}\right)\left(\rho_{j-3}^{n+1 / 2}-3 \rho_{j-1}^{n+1 / 2}+3 \rho_{j+1}^{n+1 / 2}-\rho_{j+3}^{n+1 / 2}\right)\right] .
\end{aligned}
$$

This has $|\lambda|=1$ for $\mu \leqq 2$ and the phase shift is

$$
\phi_{3}= \pm 2 \sin ^{-1}\left[\frac{1}{2} \mu \sin \theta\left\{6+\left(1-\frac{1}{4} \mu^{2}\right) \sin ^{2} \theta\right\}\right] \text {. }
$$


The limiting values of $\phi_{3} / \phi_{0}$ as $\mu \rightarrow 0$ are displayed in the second column of Table 2 : the dispersion is noticeably less than for second order schemes.

Combined fourth order scheme. A slightly more accurate formula can be devised by combining the two second order schemes described above. Then

$$
\rho_{j}^{n+1}=\rho_{j}{ }^{n}-\frac{\frac{1}{2} \mu}{1-\frac{1}{2} \mu M}\left[(1+2 M)\left(\rho_{j+1}^{n+1 / 2}-\rho_{j-1}^{n+1 / 2}\right)-M\left(\rho_{j+2}^{n}-\rho_{j-2}^{n+1}\right)\right]
$$

where

$$
M=\frac{\left(1-\frac{1}{2} \mu\right)}{6\left(1+\frac{1}{2} \mu\right)} .
$$

Once again, $|\lambda|=1$ for $\mu \leqq 2$, while the phase shift is given by

$$
\phi_{4}=-2 \tan ^{-1} \frac{(P Q-R S)}{(P S-Q R)}
$$

where

$$
\begin{array}{ll}
P=1-\mu M \sin ^{2} \theta, & Q=\frac{1}{2} \mu(1+2 M) \sin \theta \\
R=\frac{1}{2} \mu M \sin 2 \theta, & S= \pm\left[1-\frac{1}{2} \mu\left\{\frac{1}{2} \mu(1+4 M)+4 M\right\} \sin ^{2} \theta\right]^{1 / 2} .
\end{array}
$$

As $\mu \rightarrow 0$, the ratio $\phi_{4} / \phi_{0}$ tends to

$$
\frac{\mu \sin \theta(4-\cos \theta)}{3 \theta}
$$

which is listed in Table 2. The improved accuracy is evident in Figure 4.

4. Integral Formulation and Conservative Difference Schemes. The difference schemes discussed in the last section can all be expressed in conservative form. In order to do so, it is convenient to express the differential equation in terms of the integral formulatin of (1.7). This approach facilitates the expression of finite differences in more than one dimension and on curvilinear meshes. For the remainder of this paper we shall adhere to this integral formulation in order to express the conservation laws more clearly: the fundamental quantities $\rho_{j}$ and $u_{j}$ will be regarded as appropriate averages rather than values of $\rho$ and $u$ at the point $x_{j}$. In one dimension, $\rho_{j}{ }^{n}$ represents the average value ${ }^{6}$ of $\rho$ over a distance $2 \Delta x$ at the time $t^{n}$ :

$$
\rho_{j}^{n}=\frac{1}{2 \Delta x} \int_{j-1}^{j+1} \rho\left(x, t^{n}\right) d x
$$

The velocity $u$ may also vary with position (so the flow will no longer be regarded as incompressible). Following (1.7), we can express the increment in $\rho_{j}$ in terms of fluxes across the boundaries at $x_{j_{ \pm 1}}$ :

$$
\left(\rho_{j}{ }^{n+1}-\rho_{j}{ }^{n}\right) \cdot 2 \Delta x=-\left(F_{j+1}^{x}-F_{j-1}^{x}\right) \cdot \Delta t .
$$

Here $F_{k}{ }^{x} \cdot \Delta t$ represents the total mass flow (in the positive $x$-direction) across the interface at $x_{k}$ during the time interval $\left(t^{n}, t^{n+1}\right)$. Thus the fluxes $F_{k}{ }^{x}$ are time aver-

6 This definition is chosen for use on a staggered mesh. For two-level schemes it implies that $\rho$ is defined only at each alternate mesh point. 
ages at precise points in space, whereas the densities $\rho_{j}{ }^{n}$ are space averages at a precise time.

We can regard (4.2) as expressing the change in mass of a "box" centred at $x_{j}$ in terms of the fluxes of matter across its boundaries. These boxes can be combined to build up volumes; then conservation demands that the increase in mass of any such volume should equal the flow of matter into it. In one dimension, exact conservation merely requires that the flux out of the box centred on $x_{j}$, across the interface at $x_{j+1}$, be equal to the flux across that interface and into the box about $x_{j+2}$.

Now the equation (4.2) is exact: in a difference formulation we represent the fluxes in terms of the values $\rho_{j}{ }^{n}$ etc. and thereby introduce truncation errors. For

$$
F_{j}^{x}=u_{j} \rho_{j}^{x}
$$

where $\rho_{j}^{x}\left(\right.$ like $\left.F_{j}^{x}\right)$ is a spot value at $x_{j}$ but a time average over $\left(t^{n}, t^{n+1}\right)$ :

$$
\rho_{j}^{x}=\frac{1}{\Delta t} \int_{n}^{n+1} \rho\left(x_{j}, t\right) d t .
$$

These quantities are "contracted" in the $x$-direction relative to the averages $\rho_{j}{ }^{n}$ etc. from which they must be estimated. A Taylor expansion in space and time gives

$$
\begin{aligned}
\rho_{j}^{x}=\frac{1}{3}\left[4 \rho_{j}^{n+1 / 2}-\frac{1}{2}\left(\rho_{j+1}^{n}+\rho_{j-1}^{n+1}\right)\right] & \\
& -\frac{1}{6} \Delta t\left(\Delta x \frac{\partial^{2} \rho}{\partial t \partial x}-\frac{1}{2} \Delta t \frac{\partial^{2} \rho}{\partial t^{2}}\right)+O\left(\Delta x^{4}\right)
\end{aligned}
$$

or

$$
\rho_{j}^{x}=\frac{1}{12}\left[13 \rho_{j}^{n+1 / 2}-\frac{1}{2}\left(\rho_{j+2}^{n+1 / 2}+\rho_{j-2}^{n+1 / 2}\right)\right]-\frac{1}{24} \Delta t^{2} \frac{\partial^{2} \rho}{\partial t^{2}}+O\left(\Delta x^{4}\right) .
$$

Second and fourth order conservative difference schemes can be derived from these expressions.

Second order schemes. The scheme (2.5) is conservative but unstable and the onesided scheme (2.8), though stable, is not conservative. However, schemes that are properly centred can always be expressed in conservative form. The angled derivative scheme (3.1) can be reformulated in these terms if we put

$$
F_{j}^{x}=\frac{1}{2} u_{j}\left(\rho_{j-1}^{n+1}+\rho_{j+1}^{n}\right)
$$

which (see Figure 3(a)) is clearly a first order approximation to (4.3). Similarly, the staggered mesh formula (3.9) can be rewritten with

$$
F_{j}^{x}=u_{j} \rho_{j}{ }^{n+1 / 2}
$$

to achieve the same order of accuracy. Conservation then applies separately to the two sets of boxes centred on $x_{j}, x_{j \pm 2} \cdots$ and on $x_{j \pm 1}, x_{j \pm 3} \cdots$.

Fourth order schemes. In a two dimensional calculation, halving the time step doubles the computation time, while halving the mesh interval increases it eightfold. Thus it is more practicable to diminish the ratio $\Delta t / \Delta r$ than it is to decrease the interval $\Delta r$ itself. The time step must in any case be chosen to keep $\mu$ within the limit for stability; we shall impose the further restriction that $\Delta t$ be small enough to make 
$\mu^{2} \ll 1$. When $\mu \rightarrow 0$ the error $\epsilon_{j}^{n}$ defined by (2.3) depends only on $\Delta r$; we call a difference scheme accurate to order $p$ in the mesh interval $\Delta r$ if after one time step

$$
\left|\epsilon_{j}{ }^{1}\right| \leqq O\left(\Delta t \Delta x^{P}\right) \quad \text { as } \quad \frac{\Delta t}{\Delta x} \rightarrow 0 .
$$

The difference schemes we shall describe are accurate to fourth order in $\Delta x$ but only to second order in $\Delta t$. The significance of this will be discussed below.

From Equation (4.6) we can express the flux as

$$
F_{j}^{x}=\frac{1}{12} u,\left[13 \rho_{j}^{n+1 / 2}-\frac{1}{2}\left(\rho_{j+2}^{n+1 / 2}+\rho_{j-2}^{n+1 / 2}\right)\right] .
$$

This leads to a difference scheme which (when $u_{j}$ is constant) has $|\lambda|=1$ for $|\mu| \leqq 7 / 12$ and corresponds to (3.13) when $\mu^{2} \ll 1$. The dispersion has already been shown in Table 2 .

The scheme based on (4.5) is more satisfactory. This gives

$$
F_{j}^{x}=\frac{1}{3} u_{j}\left[4 \rho_{j}^{n+1 / 2}-\frac{1}{2}\left(\rho_{j+1}^{n}+\rho_{j-1}^{n+1}\right)\right] .
$$

In order to eliminate the error term of order $\Delta t \Delta x$, the direction of integration must reverse with each time step, i.e. (4.11) should alternate with

$$
F_{j}^{x}=\frac{1}{3} u_{j}\left[4 \rho_{j}^{n+1 / 2}-\frac{1}{2}\left(\rho_{j+1}^{n+1}+\rho_{j-1}^{n}\right)\right] .
$$

If $u$ is constant, $|\lambda|=1$ for $-2 \leqq \mu \leqq 6 / 5$ and (4.11) is equivalent to (3.15) with $M=1 / 6$. In Table 3 the dispersion obtained with this fourth order scheme is tabulated as a function of $\mu$ and $\theta$; it is apparent that the accuracy is not substantially impaired even if $\mu=1 / 2$. The computation illustrated in Figure 5(b) shows the improvement produced by adopting this fourth order scheme.

There are two reasons for demanding greater accuracy in $\Delta x$ than in $\Delta t$. The higher time derivatives could be estimated by substituting from the differential equation (in which case (4.11) and (4.12) would lead to (3.13) and (3.15) respectively); however, this procedure becomes indecently complicated in more than one dimension. Secondly, the error caused by terms of order $\Delta t^{2}$ in (4.5) and (4.6) is insignificant even for moderately large values of $\mu$ (as can be seen from Table 3 ). The ratio of the term of order $\Delta t^{2}$ to that of order $\Delta x^{4}$ in the error is $5 / 2(\mu / \theta)^{2}$. The error caused by setting $\mu=1 / 4$, for instance, is negligible unless $\theta \leqq 20^{\circ}$; but the whole difference

Table 3

Velocity Dispersion for Combined Fourth Order Scheme

\begin{tabular}{r|c|c|c|c|c}
\hline & \multicolumn{5}{|c}{$\mu$} \\
\cline { 2 - 6 } & 0.05 & 0.25 & 0.50 & 0.75 & 1.00 \\
\hline $18^{\circ}$ & 1.000 & 1.001 & 1.007 & 1.016 & 1.029 \\
$30^{\circ}$ & .998 & 1.002 & 1.016 & 1.039 & 1.073 \\
$45^{\circ}$ & .989 & .996 & 1.021 & 1.064 & 1.131 \\
$60^{\circ}$ & .965 & .975 & 1.005 & 1.062 & 1.156 \\
$90^{\circ}$ & .849 & .854 & .872 & .907 & .978 \\
$120^{\circ}$ & .620 & .616 & .618 & .616 & .618 \\
$180^{\circ}$ & 0 & 0 & 0 & 0 & 0 \\
\hline
\end{tabular}



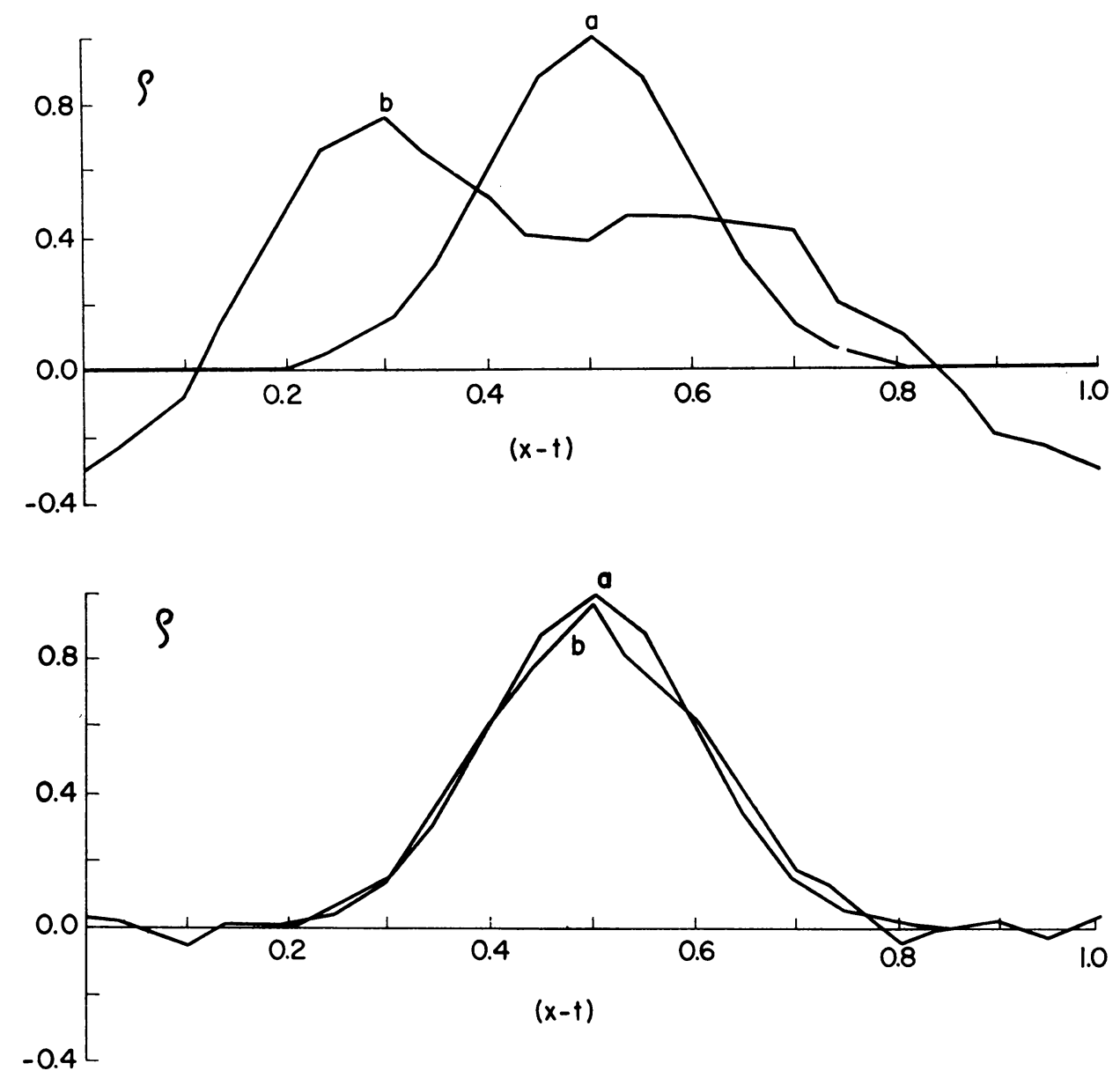

Figure 5. The effects of dispersion. The convection of a Gaussian profile with unit velocity on a mesh with $\Delta x=1 / 20, \mu=0.25$. Curves $a$ and $b$ show profiles at $t=0.0$ and $t=4.0 \mathrm{re}$ spectively. (a) Second order scheme. (b) Fourth order scheme.

scheme is bound to be sufficiently accurate for such long wave lengths. It is only in dealing with short wave lengths $\left(\theta \geqq 60^{\circ}\right)$ that the method becomes inadequate and there the effects of a finite time step are wholly unimportant.

5. Convection of a Scalar in Two Dimensions. The one dimensional problem discussed so far has been physically trivial, although it served as an adequate vehicle for introducing numerical techniques. In two dimensions, interesting problems can be posed even when div $\mathbf{u}=0$. The Eulerian difference schemes required are straightforward but careful extensions of those described already. (The advantage of using Eulerian schemes is that they can be extended to equations such as (1.3) which do not have a simple Lagrangian solution.)

Once again, we express the differential equation

$$
\frac{\partial \rho}{\partial t}=-\nabla \cdot(\rho \mathbf{u})
$$


in integral form as

$$
\Delta\left[\int_{s} \rho d S\right]=-\int \oint \rho U_{n} d l d t
$$

where $U_{n}$ is the component of velocity normal to the line element $d l$ bounding the area $S$. Let us, for the present, adopt a uniform rectangular mesh with intervals $\Delta x, \Delta y$, so that mesh points have coordinates $\left(x_{j}, y_{k}\right)=(j \Delta x, k \Delta y)$. Then we define the stored quantity $\rho_{j, k}^{n}$ as the average value of $\rho$ over a box with sides $2 \Delta x, 2 \Delta y$ centred on $\left(x_{j}, y_{k}\right)$, at the instant $t^{n}$ :

$$
\rho_{j, k}^{n}=\frac{1}{4 \Delta x \Delta y} \int_{k-1}^{k+1} \int_{j-1}^{j+1} \rho\left(x, y, t^{n}\right) d x d y .
$$

If the velocity $\mathbf{u}=(u, v)$ is steady, we define the stored components $u_{j, k}$ and $v_{j, k}$ as the average flows across line elements of length $2 \Delta y, 2 \Delta x$ respectively, centred at the point $\left(x_{j}, y_{k}\right)$ :

$$
u_{j, k}=\frac{1}{2 \Delta y} \int_{k-1}^{k+1} u\left(x_{j}, y\right) d y ; \quad v_{j, k}=\frac{1}{2 \Delta x} \int_{j-1}^{j+1} v\left(x, y_{k}\right) d x .
$$

Then the condition div $\mathbf{u}=0$ for incompressible flow, expressed as

$$
\oint U_{n} d l=0
$$

in two dimensions, has the exact finite difference analogue

$$
2 \Delta y\left(u_{j+1, k}-u_{j-1, k}\right)+2 \Delta x\left(v_{j, k+1}-v_{j, k-1}\right)=0,
$$

i.e. the net flow out of each mesh box is zero.

The fundamental difference formula, corresponding to (4.2) is

$$
\begin{array}{rl}
\left(\rho_{j, k}^{n+1}-\rho_{j, k}^{n}\right) \cdot 4 \Delta x & x y \\
& =-\left[\left(F_{j+1, k}^{x}-F_{j-1, k}^{x}\right) \cdot 2 \Delta y+\left(F_{j, k+1}^{y}-F_{j, k-1}^{y}\right) \cdot 2 \Delta x\right] \cdot \Delta t
\end{array}
$$

where

$$
\begin{aligned}
& F_{j, k}^{x}=\frac{1}{2 \Delta t \Delta y} \int_{n}^{n+1} \int_{k-1}^{k+1} \rho\left(x_{j}, y, t\right) u\left(x_{j}, y, t\right) d y d t, \\
& F_{j, k}^{y}=\frac{1}{2 \Delta t \Delta x} \int_{n}^{n+1} \int_{j-1}^{j+1} \rho\left(x, y_{k}, t\right) v\left(x, y_{k}, t\right) d x d t .
\end{aligned}
$$

Now (5.8) expresses the fluxes as the average values of products. We can re-express them as the products of averages:

$$
\begin{aligned}
& F_{j, k}^{x}=\rho_{j, k}^{x} u_{j, k}+\frac{1}{3} \Delta y^{2} \frac{\partial \rho}{\partial y} \frac{\partial u}{\partial y}+O\left(\Delta y^{4}\right), \\
& F_{j, k}^{y}=\rho_{j, k}^{y} v_{j, k}+\frac{1}{3} \Delta x^{2} \frac{\partial \rho}{\partial x} \frac{\partial v}{\partial x}+O\left(\Delta x^{4}\right) .
\end{aligned}
$$

Here $\rho_{j, k}^{x}$ and $\rho_{j, k}^{y}$ represent one-dimensional averages, contracted relative to $\rho_{j, k}^{n+12 /}$ in the $x$ and $y$ directions respectively:

$$
\begin{aligned}
& \rho_{j, k}^{x}=\frac{1}{2 \Delta t \Delta y} \int_{n}^{n+1} \int_{k-1}^{k+1} \rho\left(x_{j}, y, t\right) d y d t, \\
& \rho_{j, k}^{y}=\frac{1}{2 \Delta t \Delta x} \int_{n}^{n+1} \int_{j-1}^{j+1} \rho\left(x, y_{k}, t\right) d x d t .
\end{aligned}
$$


These quantities must be so estimated in terms of the stored values of $\rho$ as to produce a stable, conservative and accurate process. The first necessity is to determine the optimum space-time mesh. It is essential that a value of $\rho$ be available at the centre of each side of the box about a point. This restriction rules out the most obvious staggered mesh, in which the points at $t^{n+1 / 2}$ would lie at the vertices of the boxes surrounding points at time $t^{n}$. For the first estimates of $\rho_{j, k}^{x}$ and $\rho_{j, k}^{y}$ would then be the means of values at adjacent vertices, e.g. $\rho_{j, k}^{x}=\frac{1}{2}\left(\rho_{j, k+1}^{n+1 / 2}+\rho_{j, k-1}^{n+1 / 2}\right)$ and the amplitude of a Fourier mode with wave number $k_{y}$ would be multiplied by $\cos \left(k_{y} \Delta y\right)$, which would lead to serious inaccuracies even for moderately large wave lengths. Averaging may only be carried out along the direction in which a quantity has been contracted.

The optimum mesh is illustrated in Figure 6; it may be derived as follows. Suppose that (5.7) is being used to calculate $\rho_{j, k}^{n+1}$ from $\rho_{j, k}^{n}$. All the requisite values of $u, v$ and $\rho$ at $t^{n+1 / 2}$ (in particular, $\rho_{j \pm 1, k}^{n+1 / 2}$ and $\rho_{j, k \pm 1}^{n+1 / 2}$ ) must be available. Then conservation will apply over the net of points including $\rho_{j, k}^{n}$ and labelled $A$ in Figure 4 . The calculation requires the sets $C$ and $D$ of points at $t^{n+1 / 2}$; in order to advance their values to $t^{n+3 / 2}$ the set of points $B$ will be required at $t^{n+1}$. Conservation applies separately to these but they are only coupled to mesh $A$ through the sets $C$ and $D$ at the intermediate time level. There is thus a rectangular grid, with alternate points at different time levels; but this grid is made up of four independent subsidiary meshes, each with spacing $2 \Delta x, 2 \Delta y$, on which the mass is separately conserved. Correspondingly, the solution of (5.6) represents four separate flows. A slight diffusion is desirable to keep these meshes all in step (see \$7).

Second order schemes can be obtained as before. The angled derivative method gives

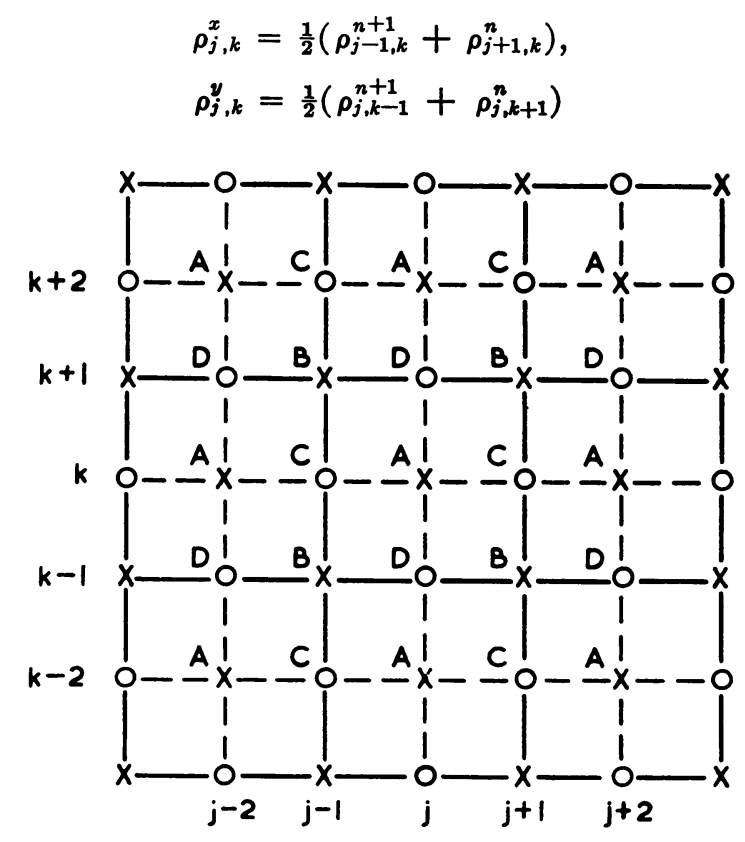

Figure 6. Staggered mesh in two dimensions. Points marked with crosses represent the meshes $A$ and $B$ at integral times $t^{n}$; points denoted by circles are on meshes $C$ and $D$ at times $t^{n+1 / 2}$. 
and has $|\lambda|=1$. Similarly, we have (corresponding to (4.8)) the staggered mesh scheme with

$$
\rho_{j, k}^{x}=\rho_{j, k}^{y}=\rho_{j, k}^{n+1 / 2} .
$$

This has $|\lambda|=1$ provided that

$$
\left|\frac{u \Delta t}{\Delta x}\right|+\left|\frac{v \Delta t}{\Delta y}\right| \leqq 2
$$

which is just the Courant-Friedrichs-Lewy criterion.

Once again, these methods can be combined to give a scheme that is accurate to fourth order in $\Delta x$ and $\Delta y$. The one-dimensional averages $\rho_{j, k}^{x}$ and $\rho_{j, k}^{y}$ can be expressed in terms of the stored mean values by

$$
\begin{aligned}
& \rho_{j, k}^{x}=\frac{1}{6}\left[8 \rho_{j, k}^{n+1 / 2}-\left(\rho_{j-1, k}^{n+1}+\rho_{j+1, k}^{n}\right)\right], \\
& \rho_{j, k}^{y}=\frac{1}{6}\left[8 \rho_{j, k}^{n+1 / 2}-\left(\rho_{j, k-1}^{n+1}+\rho_{j, k+1}^{n}\right)\right] .
\end{aligned}
$$

These have the same form as in one dimension; once again, the direction of integration should be alternated with each time step. Simpler approximations are adequate for the first derivatives in (5.9); we therefore write

$$
\begin{aligned}
F_{j, k}^{x}=\frac{1}{6}\left[u _ { j , k } \left\{8 \rho_{j, k}^{n+1 / 2}-\left(\rho_{j-1, k}^{n+1}+\right.\right.\right. & \left.\left.\rho_{j+1, k}^{n}\right)\right\} \\
& \left.+\frac{1}{8}\left(\rho_{j, k+2}^{n+1 / 2}-\rho_{j, k-2}^{n+1 / 2}\right)\left(u_{j, k+2}-u_{j, k-2}\right)\right]
\end{aligned}
$$

etc. and substitute into (5.7). The complete scheme is given in the appendix.

If $\mathbf{u}$ is independent of position, $|\lambda|=1$ provided that

$$
-2 \leqq \frac{u \Delta t}{\Delta x}+\frac{v \Delta t}{\Delta y} \leqq \frac{6}{5} .
$$

The effects of dispersion in two dimensions are illustrated in Figure 7, which shows the distortion of a circular hump as it is convected. The improvement effected by the fourth order scheme is very striking.

Figure 8 shows the results of a run in which the density distribution was distorted by a sheared velocity field. The higher order method is clearly more accurate in describing variations over only a few mesh intervals.

We have so far discussed only the linear problem where $\mathbf{u}$ is a known function of position but the treatment can equally be applied to nonlinear problems where $\mathbf{u}$ is a function of time which is computed from additional equations. The evaluation of the fluxes depends on how the stored values of $u$ and $v$ are defined and this will vary from problem to problem. For example, a two dimensional equation of motion solved in conservative form would lead to two dimensional momentum averages which would have to be contracted to yield the quantities required in (5.9).

Boundary conditions. These have not so far been mentioned. Faulty treatment of the boundaries may vitiate the computation but the proper approach is generally straightforward to establish. The simple problem of incompressible flow in a rectangular region with rigid boundaries illustrates this point.

The boundary condition is that the normal component of the velocity must vanish or, in integral form, that there is no flux across the boundary. Although div $\mathbf{u}$ vanishes if the fluid is regarded as a continuum, the finite difference analogue will 

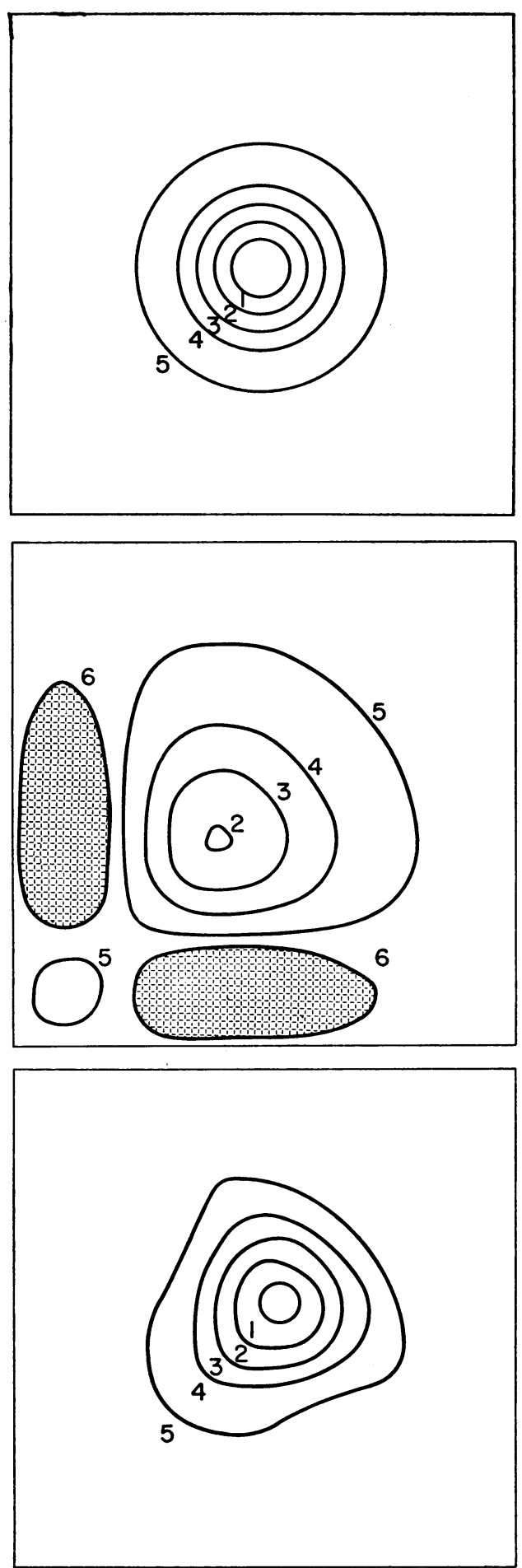

FIGURE 7. Dispersion in two dimensions: convection of a scalar by a constant velocity $\mathbf{u}=(1,1)$ on a mesh with $\Delta x=\Delta y=1 / 20, \Delta t=1 / 80$. The diagrams show contours of the scalar $\rho$ at values of $0.85,0.65,0.45,0.25,0.05$, and -0.05 , numbered from 1 to 6 respectively. (a) The original distribution; a Gaussian hump, which should be maintained as the motion is followed. (b) The distribution at $t=1.5$ (when the hump has been transported a distance $3 \sqrt{2}$ ) calculated with a second-order scheme on a staggered mesh. The hump is distorted, its height has been diminished by $30 \%$ and negative values of $\rho$ have appeared in the shaded regions. (c) The same but calculated by the fourth order scheme. The height is diminished by about $10 \%$. 

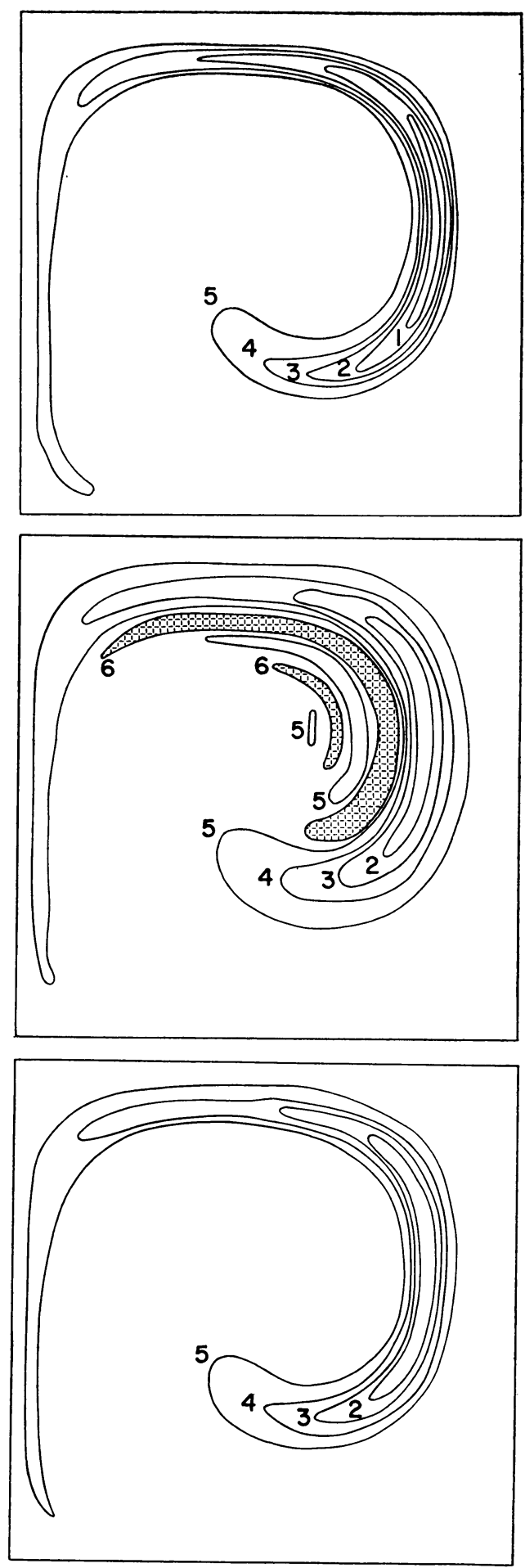

Figure 8. Convection of a scalar by a sheared velocity in two dimensions. The velocity corresponds to a stream function $-1 / \pi \sin \pi x \sin \pi y$. Initially, there was a Gaussian hump at the point $(3 / 4,3 / 4)$ which has been drawn out by the shear in the velocity. Notation as in Figure 7. (a) Distribution of $\rho$ at $t=3.0$ (calculated by fourth order scheme with $\Delta x=\Delta y=$ $1 / 100, \Delta t=1 / 200$ to give accurate results). (b) The same, calculated by a second order scheme on a staggered mesh with $\Delta x=\Delta y=1 / 50, \Delta t=1 / 100$. The maximum value is reduced by $25 \%$ and negative values appear in the shaded regions. (c) As (b) but calculated by the fourth order method. The maximum value has been reduced by less than $20 \%$. 


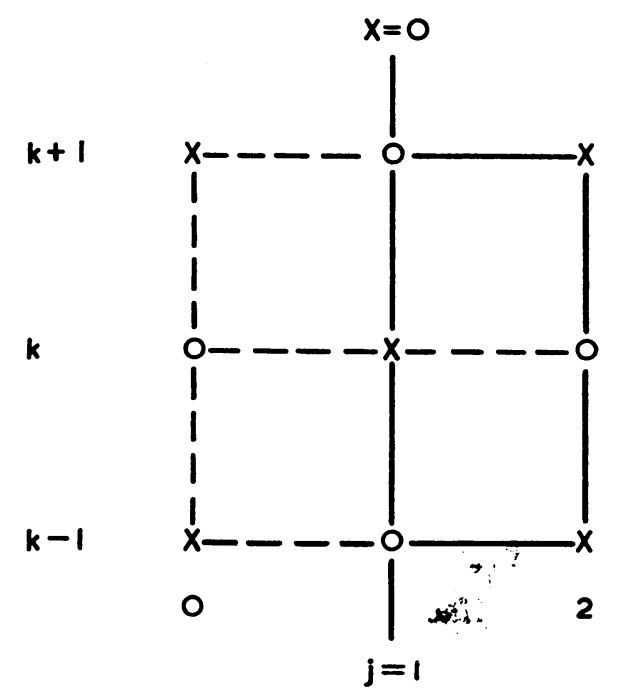

Figure 9. Treatment of the boundary.

not necessarily hold. Consider the box surrounding a point $\left(x_{1}, y_{k}\right)$ on the boundary, where $u_{1, k}=0$ (see Figure 9 ). Then the total flux into this box is

$$
F=\Delta x\left\{[\rho v]_{1, k-1}-[\rho v]_{1, k+1}\right\}-2 \Delta y\left\{[\rho u]_{2, k}\right\} .
$$

If the flux in the $y$-direction were multiplied by $2 \Delta x$ instead, then the analogue of $\operatorname{div} \mathbf{u}=0$ would not hold and mass would accumulate in (or disappear from) the box because of this numerical error. In practice, it is easier to program the computation by introducing fictitious points $\left(x_{0}, y_{k}\right)$ outside the boundary, setting $u_{o, k}$ to zero and halving the stored values of $v_{1, k}$ : then points on the boundary can be processed as interior points. Values of $\rho_{1, k}$ must be regarded as averages over boxes of area $2 \Delta x \Delta y$ centred on $\left(x_{3 / 2}, y_{k}\right)$. Boundaries parallel to the $x$-axis can be treated similarly. Analogous precautions must be taken when there is a discontinuity in the velocity within the region.

6. Convection of a Vector in Two Dimensions. In this section we consider the differential equation

$$
\frac{\partial \mathbf{B}}{\partial t}=-\operatorname{curl} \mathbf{E}=\operatorname{curl}(\mathbf{u} \wedge \mathbf{B})
$$

governing a magnetic field in a perfectly conducting fluid. In two dimensions, $\mathbf{B}$ is described by a stream function ( $z$-component of the vector potential) $\Psi$ such that

$$
\mathbf{B}=\left(\frac{\partial \Psi}{\partial y},-\frac{\partial \Psi}{\partial x}\right)
$$

Then

$$
\frac{\partial \Psi}{\partial t}=-\mathbf{u} \cdot \nabla \Psi
$$


which can be solved by the techniques of $\$ 5^{\circ}$. Moreover, lines of force are simply contours of $\Psi$ and can easily be plotted. Nevertheless, it is profitable to consider methods for integrating (6.1) directly in two dimensions, since these can be extended to three dimensional computations (where use of the vector potential does not simplify the problem). We have compared fields computed to the same order of accuracy from (6.1) and (6.2) and found them to agree.

We write (6.1) in integral form as

$$
\Delta \int_{\mathbf{S}} \mathbf{B} \cdot d \mathbf{S}=-\int \oint_{\mathbf{E}} \cdot d \mathbf{l} d t
$$

The numerical treatment of the curl in (6.1) is precisely analogous to that of the divergence in (5.1): as the divergence was expressed in terms of fluxes across surfaces, so the curl is given by circulations around the boundaries of an element of area. Then it is only necessary to estimate the relevant quantities appropriately and to ensure that over a closed surface

$$
\int \mathbf{B} \cdot d \mathbf{S}=\mathbf{0}
$$

at all times, in a finite difference form analogous to (5.6).

Let

$$
\mathbf{B}=(G, H) .
$$

The stored values of $G$ and $H$ are defined as average fluxes across the surfaces of boxes assumed to extend for unit distance in the $z$-direction:

$$
G_{j, k}^{n}=\frac{1}{2 \Delta y} \int_{k-1}^{k+1} G\left(x_{j}, y, t^{n}\right) d y \quad \text { and } H_{j, k}^{n}=\frac{1}{2 \Delta x} \int_{j-1}^{j+1} H\left(x, y_{k}, t^{n}\right) d x \text {. }
$$

In order to express (6.3) in finite difference form, we need to estimate $E_{j, k}^{\boldsymbol{z}}$, the average value over a time step of the $z$-component of $\mathbf{E}$ at the point $\left(x_{j}, y_{k}\right)$. Then

$$
\begin{aligned}
\left(G_{j, k}^{n+1}-G_{j, k}^{n}\right) \cdot 2 \Delta y & =-\left(E_{j, k+1}^{z}-E_{j, k-1}^{z}\right) \cdot \Delta t, \\
\left(H_{j, k}^{n+1}-H_{j, k}^{n}\right) \cdot 2 \Delta x & =\left(E_{j+1, k}^{z}-E_{j-1, k}^{z}\right) \cdot \Delta t .
\end{aligned}
$$

The electric fields $E_{j, k}^{z}$ represent spot values of the $z$-component of $-(\mathbf{u} \wedge \mathbf{B})$ and can be written

$$
E_{j, k}^{z}=\left(u_{j, k}^{y} H_{j, k}^{x}-v_{j, k}^{x} G_{j, k}^{y}\right) .
$$

As in (5.10), the superscript letter indicates the direction in which an average has been contracted; thus the quantities on the right-hand side of (6.9) are spot values at the point $\left(x_{j}, y_{k}\right)$ and, in the case of the magnetic field, averages over one time step:

$$
G_{j, k}^{y}=\frac{1}{\Delta t} \int_{n}^{n+1} G\left(x_{j}, y_{k}, t\right) d t \quad \text { and } H_{j, k}^{x}=\frac{1}{\Delta t} \int_{n}^{n+1} H\left(x_{j}, y_{k}, t\right) d t .
$$

(The velocity is assumed to be steady and independent of time.) The field components in (6.9) must be estimated in terms of the stored averages $G_{j, k}^{n}$ and $H_{j, k}^{n}$.

Only one of (6.7) and (6.8) need be solved; the other component can then be 
obtained from (6.4). If we consider the flux out of the box centred at $\left(x_{j}, y_{k}\right)$ then $H_{j, k+1}^{n+1}$ could be evaluated from (6.8) and $\mathrm{G}_{j+1, k}^{n+1}$ from

$$
\left(H_{j, k+1}^{n+1}-H_{j, k-1}^{n+1}\right) \cdot 2 \Delta x+\left(G_{j+1, k}^{n+1}-G_{j-1, k}^{n+1}\right) \cdot 2 \Delta y=0 .
$$

This procedure automatically ensures that the finite difference analogue of $\operatorname{div} \mathbf{B}=\mathbf{0}$ is satisfied.

Second order schemes. Consider the box centred on $\left(x_{j}, y_{k}\right)$ in Figure 6. For a two level scheme, $G$ must be defined at the points of mesh $C$ and $H$ at those of mesh $D$. For the angled derivative method we set

$$
G_{j+1, k+1}^{y}=\frac{1}{2}\left(G_{j+1, k}^{n+1}+G_{j+1, k+2}^{n}\right), \quad H_{j+1, k+1}^{x}=\frac{1}{2}\left(H_{j, k+1}^{n+1}+H_{j+2, k+1}^{n}\right)
$$

etc. Suppose that the sweep proceeds over increasing values of $y$ for each value of $x$ and that we integrate $H$ but set $G$ from (6.11). Then we know all the quantities required for calculating $H_{j, k+1}^{n+1}$ except $G_{j+1, k}^{n+1}$. But this value is known implicitly from (6.11) and we can thus simultaneously calculate $G_{j+1, k}^{n+1}$ and $H_{j, k+1}^{n+1}$.

On a staggered mesh, we simply take

$$
G_{j, k}^{y}=G_{j, k}^{n+1 / 2} ; \quad H_{j, k}^{x}=H_{j, k}^{n+1 / 2} .
$$

The mesh is basically the same as that used for the scalar equation but the disposition of quantities over the points is more complicated. There are in fact four separate meshes: $A^{\prime}$ and $B^{\prime}$ at integral time levels and $C^{\prime}$ and $D^{\prime}$ at intermediate levels. In each of these, $G, H, u$ and $v$ are defined at points of the meshes in Figure 4, as summarized in Table 4. All quantities $u, v, G$ and $H$ are defined at each alternate point in space on a given time level. However, conservation of flux in the form (6.11) applies only to the individual meshes $A^{\prime}, B^{\prime}, C^{\prime}$ and $D^{\prime}$. Moreover, the two meshes at a given time level are only related indirectly, via those at the intermediate level.

Fourth order scheme. The same staggered mesh can be used and the procedure follows that outlined for the angled derivative method. Thus, if we sweep over $y$ for each value of $x$, new values of $H$ may be found by integration while those of $G$ are calculated from the flux conservation law (6.11). In (6.9) we set

$$
\begin{aligned}
G_{j, k}^{y} & =\frac{1}{6}\left[8 G_{j, k}^{n+1 / 2}-\left(G_{j, k-1}^{n+1}+G_{j, k+1}^{n}\right)\right], \\
H_{j, k}^{x} & =\frac{1}{6}\left[8 H_{j, k}^{n+1 / 2}-\left(H_{j-1, k}^{n+1}+H_{j+1, k}^{n}\right)\right],
\end{aligned}
$$

and substitute into (6.8). Before we can evaluate $H_{j, k+1}^{n+1}$ (and, from it, $G_{j+1, k}^{n+1}$ as well) it proves necessary to express $G_{j-1, k+1}^{n}$ and $G_{j+1, k}^{n+1}$ in terms of values of $G$ and $H$ that are currently available, by using (6.11). The full difference formula appears in the appendix. Once again, the directions of integration should be reversed after each time step.

Integral formulation is more important for the vector than for the scalar equation: it leads naturally and unambiguously to two equivalent expressions ${ }^{7}$ with fourth order accuracy in $\Delta x$ and $\Delta y$, in which only four spot values of the velocity components appear. Moreover, flux is exactly conserved. By contrast, a fourth order treatment of $(6.1)$ in the differential forms

$$
\frac{\partial H}{\partial t}=-\frac{\partial}{\partial x}(u H-v G)
$$

${ }^{7}$ We could have chosen to integrate $G$ and to set $H$ from $\operatorname{div} B=0$. 
TABLE 4

Defining Quantities on a Staggered Mesh in Two Dimensions

\begin{tabular}{c|c|c|c|c|c}
\hline Mesh & $G$ & $H$ & $u$ & $v$ & Time level \\
\hline$A^{\prime}$ & $A$ & $B$ & $B$ & $A$ & $t^{n}$ \\
$B^{\prime}$ & $B$ & $A$ & $A$ & $B$ & $t^{n}$ \\
$C^{\prime}$ & $C$ & $D$ & $D$ & $C$ & $t^{n+1 / 2}$ \\
$D^{\prime}$ & $D$ & $C$ & $C$ & $D$ & $t^{n+1 / 2}$ \\
\hline
\end{tabular}

or

$$
\frac{\partial H}{\partial t}=\mathbf{B} \cdot \boldsymbol{\nabla} v-\mathbf{u} \cdot \boldsymbol{\nabla} H-H \boldsymbol{\nabla} \cdot \mathbf{u}
$$

would have involved more values of the velocity components without ensuring exact conservation of flux. Simplifying the expressions for $\mu^{2} \ll 1$ would also have proved tricky.

In discussing these schemes, it has been supposed that values of $u_{j, k}^{y}$ and $v_{j, k}^{x}$ are already known. This will be so if they are independent of time but in a nonlinear problem spot values of the velocity components must be evaluated from the averages that are available. If density and magnetic field are being evaluated then the velocity components cannot simultaneously be appropriately defined for both of them.

The simplest boundary condition is that the normal components of both $\mathbf{u}$ and B should vanish at the walls (which must then be rigid and perfectly conducting). Then the electric field likewise vanishes and boundary points can be treated as interior points if an extra row is inserted as in Figure 9.

7. The Introduction of a Slight Diffusion. The schemes outlined above are prone to two different numerical errors, which can be remedied by including a small diffusive coefficient. First, it is quite possible for values of quantities on each of the four independent meshes to differ by constant amounts; indeed, such differences will be produced on a staggered mesh by inexact initial values. Secondly, any numerical method, subtle though it may be, is bound to fail for variations on the scale of a few mesh intervals. Yet the velocity field will usually generate such finescale effects, whose computed behaviour will then be peculiar. This can be masked by inserting diffusion into the equations so that the fine-scale variations are obliterated [6].

The differential equations describe a nonlinear coupling between Fourier modes, by which energy is continually fed into higher and higher modes. A similar coupling is described by the difference equations, except that energy which ought to be fed into very high modes may appear in lower ones instead, since modes with wave numbers $\left(k_{x}, k_{y}\right)$ and $\left(k_{x}+2 l \pi / \Delta x, k_{y}+2 m \pi / \Delta y\right)$, where $l$ and $m$ are integers, cannot be distinguished. Any difference scheme will therefore couple the wrong modes for large $k$ even if energy is conserved by the numerical process. Unless energy is removed by diffusion as it reaches these high wave numbers, physical errors will arise.

In fact, the difference schemes were developed to study the equation

$$
\frac{\partial \mathbf{B}}{\partial t}=\operatorname{curl}(\mathbf{u} \wedge \mathbf{B}-\eta \operatorname{curl} \mathbf{B})=\operatorname{curl}(\mathbf{u} \wedge \mathbf{B})+\eta \nabla^{2} \mathbf{B}
$$


under conditions when the magnetic Reynolds number $R_{m}=U L / \eta$ was very large and the time variation of $\mathbf{B}$ was slight. (Here $U$ is a characteristic speed and $L$ a length characteristic of the system; the resistivity is assumed to be uniform.) Then the curl of $(\mathbf{u} \wedge \mathbf{B})$ almost vanishes and the residue is balanced by a small diffusive term. So it is more practical to accept the presence of the resistive term ${ }^{8}$ and then to ask: how large a value of $R_{m}$ can be described adequately by the numerical process on a given mesh? A reasonable requirement is that diffusion should dominate convection for modes with a dispersion error of more than $5 \%$. On a grid of $50 \times 50$ points with a smooth velocity field, a second order scheme can treat $R_{m} \leqq 200$ and a fourth order scheme $R_{m} \leqq 1000$ with sufficient accuracy. This is confirmed in practice and the maximum value of $R_{m}$ varies as the square of the number of mesh intervals $J$. An effective lower limit to $R_{m}$ is set by assuming that the choice of $\Delta t$ is not affected by the resistivity : then $R_{m} \geqq J$.

For constant $\eta$, the resistive terms in (1.3) and (1.4) can be approximated to by the Dufort-Frankel method [1]. To the scalar equation we add terms of the form

$$
\begin{aligned}
\eta \frac{\Delta t}{\Delta x^{2}}\left[\left(\rho_{j+1, k}^{n+1 / 2}+\rho_{j-1, k}^{n+1 / 2}\right)-\left(\rho_{j, k}^{n}\right.\right. & \left.\left.+\rho_{j, k}^{n+1}\right)\right] \\
& +\eta \frac{\Delta t}{\Delta y^{2}}\left[\left(\rho_{j, k+1}^{n+1 / 2}+\rho_{j, k-1}^{n+1 / 2}\right)-\left(\rho_{j, k}^{n}+\rho_{j, k}^{n+1}\right)\right] .
\end{aligned}
$$

This method is unconditionally stable and sufficiently accurate for our purpose so long as $\eta$ is small. These resistive terms are incorporated in the formulae in the Appendix. If $\eta$ is a function of position, the second differences can simply be altered to include this variation. Resistive diffusion causes the four meshes $A^{\prime}, B^{\prime}, C^{\prime}$, and $D^{\prime}$ to relax exponentially to the same values with a time constant $\left(\Delta x^{2} / 2 \eta\right)$. Since all the meshes are connected through the diffusive terms, one might expect conservation on each mesh (and over the system as a whole) to be affected, but it can be shown that magnetic flux continues to be conserved.

8. Extension to Three Dimensions. The higher order methods introduced in $\$ 4$ extend the class of problems that can be tackled on a given mesh in two dimensions. In three dimensions, schemes with this accuracy are essential. The necessary staggered mesh is illustrated in Figure 10: it can be thought of as the crystal lattice of $\mathrm{NaCl}$ with, say, sodium atoms representing points at integral times and chlorine atoms those at half-integral time levels. Thus there are two interpenetrating face-centred cubic lattices. Values of all three components of both $\mathbf{u}$ and $\mathbf{B}$, and also that of $\rho$, have to be stored at each point on the mesh.

The necessary difference schemes, though complicated, can be constructed on exactly the same principles as those of $\$ 5$ and $\$ 6$. We put

$$
\begin{aligned}
\left(\rho_{j, k, l}^{n+1}-\rho_{j, k, l}^{n}\right) \cdot 8 \Delta x \Delta y \Delta z=-\left[\left(F_{j+1, k, l}^{x}-F_{j-1, k, l}^{x}\right) \cdot 4 \Delta y \Delta z\right. \\
\left.\quad+\left(F_{j, k+1, l}^{y}-F_{j, k-1, l}^{y}\right) \cdot 4 \Delta z \Delta x+\left(F_{j, k, l+1}^{z}-F_{j, k, l-1}^{z}\right) \cdot 4 \Delta x \Delta y\right] \cdot \Delta t
\end{aligned}
$$

and

$$
\begin{aligned}
& \left(G_{j, k, l}^{n+1}-G_{j, k, l}^{n}\right) \cdot 4 \Delta y \Delta z \\
& \quad=-\left[\left(E_{j, k+1, l}^{z}-E_{j, k-1, l}^{z}\right) \cdot 2 \Delta z-\left(E_{j, k, l+1}^{y}-E_{j, k, l-1}^{y}\right) \cdot 2 \Delta y\right] \cdot \Delta t
\end{aligned}
$$

${ }^{8}$ If the actual resistivity were negligible, an artificial fourth order diffusion term, $-\eta \nabla^{4} \mathbf{B}$, might be inserted: this would cut off the smallest modes much more steeply and tidy the calculation, provided that it left the physics unaffected. 


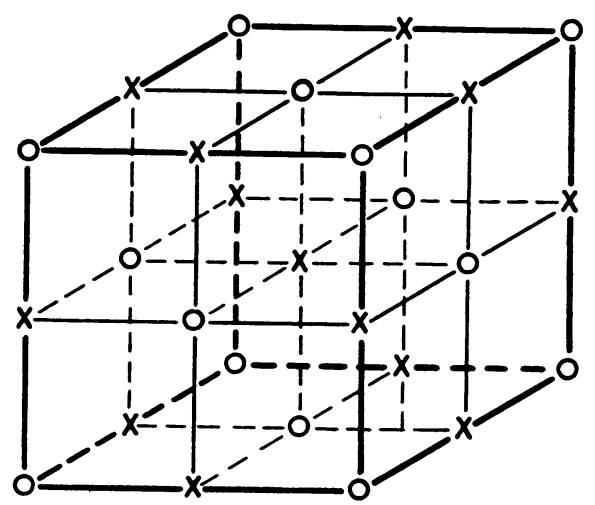

FIGURE 10. Staggered mesh in three dimensions. Circles indicate points at integral time $t^{n}$ and crosses denote those at intermediate times $t^{n+1 / 2}$.

etc. The fluxes $F^{x}, F^{y}, F^{z}$ and the electric field components $E^{x}, E^{y}$ and $E^{z}$ have then to be estimated. It should be noted that the averaging processes differ for the various components of $(\rho \mathbf{u})$ and $(\mathbf{u} \wedge \mathbf{B})$. The components of $\mathbf{u}$ and $\mathbf{B}$ themselves will be defined over surfaces while the "spot values" of (8.2) now correspond to averages along lines through these surfaces. Thus the combination taken will depend on the line that is chosen: for the normal field at the centre of a face of the cube in Figure 11 , the numerical scheme merely estimates $\Phi(\mathbf{u} \wedge \mathbf{B}): d 1$ taken around the perimeter of that face. The final expressions resemble those obtained for two dimensions and will not be given here.

The averaging process depends on how $\mathbf{u}$ has been calculated from the equation of motion. In some cases, the velocity may be a function of the field $\mathbf{B}$ and of $\rho$ and calculable from them [13]. In others it must be found from the equation of motion, which can itself be expressed in the conservative form

$$
\frac{\partial}{\partial t}(\rho \mathbf{u})=-\nabla \cdot P
$$

(where $P$ is a pressure tensor) analogous to (1.1) and (1.2). In that case the fundamental quantities are $\rho_{j, k, l}^{n}$ and

$$
(\rho \mathbf{u})_{j, k, l}^{n}=\frac{1}{8 \Delta x \Delta y \Delta z} \iiint \rho\left(x, y, z, t^{n}\right) \mathbf{u}\left(x, y, z, t^{n}\right) d x d y d z
$$

and $\mathbf{u}$ must be evaluated from them.

9. The Treatment of Polar Meshes. All that has been said so far applies equally to polar meshes. It need only be remembered that the dimensions of the opposite sides of a box are no longer identical. Fluxes and circulations must therefore be weighted accordingly. Consider, for example, polar co-ordinates $(r, \theta)$ in two dimensions. We define quantities at the points $\left(r_{j}, \theta_{k}\right)$ on a mesh with spacing $\Delta r, \Delta \theta$ and have to bear in mind that the arc lengths $\left(r_{j-1} \Delta \theta\right)$ and $\left(r_{j+1} \Delta \theta\right)$ are unequal. Thus, for example, (5.7) becomes

$$
\begin{aligned}
& \left(\rho_{j, k}^{n+1}-\rho_{j, k}^{n}\right) \cdot 4 r_{j} \Delta r \Delta \theta \\
& \quad=-\left[\left(r_{j+1} F_{j+1, k}^{r}-r_{j-1} F_{j-1, k}^{r}\right) \cdot 2 \Delta \theta+\left(F_{j, k+1}^{\theta}-F_{j, k-1}^{\theta}\right) \cdot 2 \Delta r\right] \cdot \Delta t .
\end{aligned}
$$

The equations for a magnetic field require similar alterations. 
In three dimensions, the scalar equation expressed in cylindrical polar coordinates $(r, \theta, z)$ resembles $(9.1)$ but the expressions for the magnetic field are more involved. For the $z$-component, $K$, of $\mathbf{B}$ we have

$$
\begin{aligned}
& \left(K_{j, k, l}^{n+1}-K_{j, k, l}^{n}\right) \cdot 4 r_{j} \Delta r \Delta \theta \\
& \quad=-\left[\left(r_{j+1} E_{j+1, k, l}^{\theta}-r_{j-1} E_{j-1, k, l}^{\theta}\right) \cdot 2 \Delta \theta-\left(E_{j, k+1, l}^{r}-E_{j, k-1, l}^{r}\right) \cdot 2 \Delta r\right] \cdot \Delta t
\end{aligned}
$$

The corresponding equations is spherical polar coordinates are more complicated.

10. Summary. We have throughout preferred to integrate the differential equations over elements of space-time defined by mesh points. The values of variables in the differential equations are then replaced by averages, whose definition must be borne in mind when formulating the difference schemes. This approach has enabled us to devise schemes with a high order of accuracy, in a consistent manner.

For example, the value of density stored at a point is defined as the average over a box surrounding that point, at a given time. After a time step the increment in the density is obtained by evaluating the total flux of matter into the box during that interval. Similarly, a component of magnetic field is defined as the average through an element of area at a given time. The increment in the field is then the integral of the e.m.f. around a line enclosing the element, taken over one time step.

Thus we can distinguish between fundamental quantities, which are stored, and the fluxes which change their values. The former are defined as averages over volumes or surfaces (in three dimensions) while the latter are averages over surfaces or lines and over time. Simple boundary conditions then imply that the fluxes vanish at the walls. Our problem has been to compute the fluxes from the fundamental quantities in such a way that mass and magnetic flux are exactly conserved and a sufficiently accurate solution is produced. Adequate accuracy is obtained by estimating fluxes with schemes that are correctly centred in time. This favours the adoption of a staggered mesh, where fundamental quantities are defined at the points where they are required. Making a Taylor expansion in the space coordinates enables us to choose schemes that minimize the coefficients of fourth order terms in the mesh spacing. The latest values of fundamental quantities available at each mesh point can conveniently be incorporated into partially implicit methods.

Acknowledgements. The angled derivative method was developed in collaboration with Dr. F. Hertweck. This paper owes much to discussions with Dr. K. W. Morton; we are also grateful to Mr. R. L. Parker for help in calculating the data in the tables.

\section{APPEndix.}

Fourth order difference schemes on a rectangular mesh in two dimensions. The sweep proceeds over increasing values of $j$ and $k$. We set

$$
\begin{aligned}
X & =\frac{\Delta t}{12 \Delta x} & L & =\eta \frac{\Delta t}{\Delta x^{2}} \\
Y & =\frac{\Delta t}{12 \Delta y} & M & =\eta \frac{\Delta t}{\Delta y^{2}}
\end{aligned}
$$

and write

$$
\delta_{x} \rho_{j, k}^{n+1 / 2}=\frac{1}{4}\left(\rho_{j+2, k}^{n+1 / 2}-\rho_{j-2, k}^{n+1 / 2}\right)
$$


etc. as an estimate of the first derivative. Then

$$
\rho_{j, k}^{n+1}=\frac{B \rho_{j, k}^{n}+C+D+E}{A}
$$

where

$$
\begin{aligned}
A= & 1-\left(X u_{j+1, k}+Y v_{j, k+1}\right)+(L+M) \\
B= & 1-\left(X u_{j-1, k}+Y v_{j, k-1}\right)-(L+M) \\
C= & X\left[u_{j-1, k}\left(8 \rho_{j-1, k}^{n+1 / 2}-\rho_{j-2, k}^{n+1}\right)-u_{j+1, k}\left(8 \rho_{j+1, k}^{n+1 / 2}-\rho_{j+2, k}^{n}\right)\right. \\
& \left.\quad+2\left(\delta_{y} \rho_{j-1, k}^{n+1 / 2} \cdot \delta_{y} u_{j-1, k}-\delta_{y} \rho_{j+1, k}^{n+1 / 2} \cdot \delta_{y} u_{j+1, k}\right)\right] \\
D= & Y\left[v_{j, k-1}\left(8 \rho_{j, k-1}^{n+1 / 2}-\rho_{j, k-2}^{n+1}\right)-v_{j, k+1}\left(8 \rho_{j, k+1}^{n+1 / 2}-\rho_{j, k+2}^{n}\right)\right. \\
& \left.\quad+2\left(\delta_{x} \rho_{j, k-1}^{n+1 / 2} \cdot \delta_{x} v_{j, k-1}-\delta_{x} \rho_{j, k+1}^{n+1 / 2} \cdot \delta_{x} v_{j, k+1}\right)\right] \\
E= & L\left(\rho_{j+1, k}^{n+1 / 2}+\rho_{j-1, k}^{n+1 / 2}\right)+M\left(\rho_{j, k+1}^{n+1 / 2}+\rho_{j, k-1}^{n+1 / 2}\right) .
\end{aligned}
$$

We integrate

$$
H_{j, k}^{n+1}=\frac{B^{\prime} H_{j, k}^{n}+C^{\prime}+D^{\prime}+E^{\prime}}{A^{\prime}}
$$

and set

$$
G_{j, k}^{n+1}=G_{j-2, k}^{n+1}+\frac{\Delta x}{\Delta y}\left(H_{j-1, k-1}^{n+1}-H_{j-1, k+1}^{n+1}\right)
$$

from $\operatorname{div} \mathbf{B}=\mathbf{0}$.

$$
\begin{aligned}
A^{\prime}= & 1-\left(X u_{j+1, k}^{y}+Y v_{j, k+1}^{x}\right)+(L+M) \\
B^{\prime}=1 & -\left(X u_{j-1, k}^{y}+Y v_{j, k-1}^{x}\right)-(L+M) \\
C^{\prime}=- & X\left[u_{j+1, k}^{y}\left(8 H_{j+1, k}^{n+1 / 2}-H_{j+2, k}^{n}\right)-u_{j-1, k}^{y}\left(8 H_{j-1, k}^{n+1 / 2}-H_{j-2, k}^{n+1}\right)\right. \\
& \quad-8\left(v_{j+1, k}^{x} G_{j+1, k}^{n+1 / 2}-v_{j-1, k}^{x} G_{j-1, k}^{n+1 / 2}\right) \\
& \left.\quad+\left(v_{j+1, k}^{x}-v_{j-1, k}^{x}\right)\left(G_{j-1, k-1}^{n+1}+G_{j+1, k+1}^{n+1}\right)\right] \\
D^{\prime}=- & -Y\left[v_{j+1, k}^{x} H_{j, k-2}^{n+1}-v_{j-1, k}^{x} H_{j, k+2}^{n}\right] \\
E^{\prime}= & L\left(H_{j+1, k}^{n+1 / 2}+H_{j-1, k}^{n+1 / 2}\right)+M\left(H_{j, k+1}^{n+1 / 2}+H_{j, k-1}^{n+1 / 2}\right) .
\end{aligned}
$$

U.K.A.E.A., Culham Laboratory, Abingdon, Berks

England

1. P. D. LAX, "Weak solutions of nonlinear hyperbolic equations and their numerical computation," Comm. Pure Appl. Math., v. 7, 1954, pp. 159-193. MR 16, 524.

2. P. D. Lax, "Hyperbolic systems of conservation laws. II," Comm. Pure Appl. Math., v. 10, 1957, pp. 537-566. MR 20 \#176.

3. R. D. Richtmyer, Difference Methods for Initial-Value Problems, Interscience Tracts in Pure and Applied Mathematics, No. 4, Interscience, New York, 1957. MR 20 \#438.

4. P. D. LaX \& B. Wendroff, "Systems of conservation," Comm. Pure Appl. Math., v. 13,1960 , pp. $217-237$. MR $22 \# 11523$.

5. P. D. LAX \& B. WENDROFF, "Difference schemes for hyperbolic equations with high order of accuracy," Comm. Pure Appl. Math., v. 17, 1964, pp. 381-398. MR $30 \# 722$.

6. R. D. Richtmyer, N. C. A. R. Technical Notes, 63-2, 1963. 
7. O. Johansson \& H. O. Kreiss, B. I. T., v. 3, 1963, pp. 97-107.

8. K. V. Roberts, F. HerTweCK \& S. J. RoberTs, Culham Laboratory Report, CLM-R29, 1963.

9. V. K. SAUL'Ev, Dokl. Akad. Nauk SSSR, v. 115, 1957, p. 1077.

10. J. H. Giese, "Numerical analysis," Recent Soviet Contributions to Mathematics, J. P. La Salle \& S. Lefshetz (Eds.), Macmillan, New York, 1962.

11. N. A. Phillips, "Numerical weather prediction," Advances in Computers, Vol. I, Academic Press, New York, 1960, pp. 43-90. MR 22 \#5461.

12. J. Smagorinsiy, Monthly Weather Review, v. 86, 1959, p. 457.

13. J. B. TAYLOR, Proc. Roy. Soc. Ser. A, v. 274, 1963, p. 274. 